\title{
Cases for Additive Manufacturing on the International Space Station
}

\author{
Cooper, Kenneth G., and McLemore, Carole \\ NASA Marshall Space Flight Center, Huntsville, AL 35812 \\ Anderson, Theodore "Ted" \\ Raytheon Systems, Inc, Huntsville, AL 35810
}

\begin{abstract}
There are thousands of plastic or non-structural metal components on the International Space Station (ISS), any of which could require replacing sometime between resupply missions. While these may not be life critical, it can cause significant delays to flight projects that have to wait several weeks to months to receive a key part - one that could have been designed and built on-board the ISS within a few hours. A plastic deposition additive manufacturing process is a low-energy, low-mass solution to many common needs on board the ISS.
\end{abstract}

\section{Nomenclature}

ISS $=$ International Space Station

$P D A M=$ plastic deposition additive manufacturing

ABS $=$ acrylonitrile butadiene styrene

NASA $=$ National Aeronautics and Space Administration

zero-g = short for "zero gravity", also referring to the microgravity state on-board the ISS

SLS = selective laser sintering, a powder-based additive manufacturing process

PRACA = problem resolution and corrective action database for the ISS

\section{Introduction}

A

S of 2010, the International Space Station (ISS) has been commissioned to continue orbiting the Earth to conduct science and engineering experiments for another ten years. The primary vehicles for re-supplying the station are now retired, with the last flight being completed by the Space Shuttle Atlantis in the summer of 2011. With the absence of an American space flight vehicle to send up replacement tools and parts, the United States will be reliant solely on foreign partners to carry and deliver critical goods and components. The expense of space flight coupled with the amount of unknown needs of the inhabitants of the ISS which are typically in the form of lost tools or specialty tools for unique cases and the case for having an on-board parts fabricator is multiplied.

There are thousands of plastic or non-structural metal components on the ISS to date. Any of these components could require being replaced or repaired between resupply missions. While some of these components may not be life critical, it can cause significant delays to flight projects and experiments that will have to wait several weeks or months to receive a key part or component. With an on-board parts fabricator, the part could have been designed and built on the ISS within a few hours.

One potential parts fabricator that could be put on-board the ISS is a plastic deposition additive manufacturing process. A plastic deposition additive manufacturing process is a low-energy, low-mass solution to many common needs on board the ISS. These systems "print" plastic parts by extruding thin layers of material and tracing the shape of the part from a three dimensional computer design. The final parts are not as strong or accurate as machined metal or injection-molded plastic components but they can be fabricated with a plastic deposition additive manufacturing process to get the job done nearly immediately and get an expensive flight experiment back on course.

An additional benefit to the plastic deposition additive manufacturing process is that it utilizes a plastic filament feedstock. By utilizing a filament, these systems can be utilized in a zero gravity environment. Additionally, recycling the material from previously built parts and utilizing provisioned resources like packing containers to create and replenish the plastic filament feedstock will be critical in sustaining the machines with build material.. 


\section{Background}

In 1993 the NASA Marshall Space Flight Center (MSFC) procured its first plastic deposition additive manufacturing (PDAM) machine with the intentions to develop the machine for space flight operations and other uses. The machine was an early beta-test system. NASA worked with the vendor for several years to upgrade and modify the system to handle a wider range of temperatures. The wider range of temperatures helped increase the material capabilities of the system from various types of waxes to a more durable nylon material and finally to a structural acrylonitrile butadiene styrene (ABS) thermoplastic. The successful development of the ABS material in the system made it more attractive and realistic to consider fabricating useful components onboard a spacecraft.

The NASA MSFC team partnered with two other organizations that had the similar interest to get a fabrication system in space: NASA Johnson Space Center (JSC) and the Milwaukee School of Engineering (MSOE). The first step was to acquire zero-gravity flights to prove the technology would work in a spacecraft situation. The team conducted ground experiments by rotating the PDAM equipment onto its side and putting the system upside down to demonstrate successful fabrication of components against the normal pull of gravity.

By 1999 the team had a test equipment data plan and had completed flight training. In the summer of 1999, the PDAM technology had successfully completed zero-g flights. A total of four flights were successfully conducted on NASA's KC-135 aircraft. The KC-135 aircraft flies in parabolic arcs to create a weightless environment. The total flight time was 10-12 hours with a total of one hour and twenty minutes of zero-g time. The zero-g time was exploited to fabricate several small test coupons.

During the same timeframe of the zero-g test flights, Rocketdyne (now a division of Pratt \& Whitney) successfully certified parts fabricated from a specific nylon material in the Selective Laser Sintering (SLS) additive manufacturing process to be flown and used on the International Space Station. Currently there are hundreds of parts on the ISS that were built using additive manufacturing technologies not to mention the other plastic and nonmetallic components built with traditional methods. It has been successfully shown that additive manufactured parts can be used on the ISS, so the next step is to put an additive manufacturing system on-board to create

\section{Replaceable Parts on ISS}

\begin{tabular}{|l|r|}
\hline Electrical/Electronic & $29.6 \%$ \\
\hline Plastic/Composite & $28.6 \%$ \\
\hline Mechanical/Metal & $16.5 \%$ \\
\hline Ceramics/Glass & $7.3 \%$ \\
\hline Total Replaceable & $82.0 \%$ \\
\hline
\end{tabular}

Figure 1. Potential replaceable parts on the ISS. Based on the Problem Resolution And Corresponding Action (PRACA) databased maintained by NASA on the International Space Station, about $1 / 3^{\text {rd }}$ (28.6\%) of the parts that need replaced are non-metal, in the form of gaskets, hoses, valve seals, retainer brackets and lubricants. Many of the parts may be likely candidates for additive fabrication.

replacement parts. There are multiple scenarios for which additive manufacturing will be a critical asset on a long duration mission; particularly a plastic deposition additive manufacturing (PDAM) process due to its ease of use and zero gravity friendliness:

\section{A. Case\#1: Known/Predicted Repair}

Some parts are just going to break or wear out; sometimes by design. Because these breaks are known or predicted, the logistics teams for a spacecraft can plan ahead and stockpile spares for those particular parts and institute a preventative maintenance program to replace parts before failure. However, if custom replacements could be made on-board in a just-in-time approach, these parts could be put to use till failure in non-critical situations. Thus the parts have a much longer useful life rather than replacing them on a set preventative maintenance schedule.

In addition, while the savings would essentially be mass-neutral, the volume and logistics savings of storing and tracking the actual parts versus "printing" the parts on demand from a compact spool of feedstock are much more attractive. About 1/3rd of all replacement parts currently on the International Space Station are made from nonmetallic materials, particularly plastics and rubbers that can be simulated closely with the PDAM process. SEE FIGURE 1. 


\section{B. Case\#2: Known Production \& Assembly}

Flight experiments and payloads can be designed more optimally if the investigators know that some of their hardware components can be built on-orbit. Structural and geometrical constraints caused by launch loads and vehicle stowage requirements may be by-passed in order to build components in space and taking advantage of the absence of gravity.

\section{Case\#3: Unknown Repair and Replacement}

Even with the most careful planning it is likely that we will miss carrying the proper amount of spares to cover every possible contingency scenario. For instance, tools that are lost or damaged are typically replaced in significant numbers with each resupply mission from Earth. The ability to create makeshift replacement tools while waiting for resupply could prevent flight experiments from losing critical run-time as well as possibly serving as a life saver in critical human systems equipment.

\section{Case\#4: New Experimentation Advantages}

Given the opportunity to build freeform shapes in space, researchers on the ground and on-board alike will undoubtedly discover new and interesting ways to use the technology. One such opportunity is to include educational aspects where students can design and build parts in space. The applications opportunities become virtually boundless.

\section{Conclusion}

A plastic deposition additive manufacturing machine installed on the International Space Station will provide a core capability for sustaining the outpost for its full lifetime. It will also help in the preparation for future long duration human expeditions into space. While direct metal additive manufacturing techniques will also be required for future long-term missions, the cost and mass required to launch and the technology development needed for such a process may not be feasible in the remaining years of the ISS's planned lifespan. The capability to produce parts on board the ISS will provide various benefits for educational purposes through potentially saving the ship in a dire emergency situation. 